\title{
Thymoquinone Crosstalks with DR5 to Sensitize TRAIL Resistance and Stimulate ROS-Mediated Cancer Apoptosis
}

\author{
Ahmed A Abd-Rabou ${ }^{1 *}$, Nagwa M Abd El-Salam², Hayat M I Sharada ${ }^{3}$, Gehan \\ G Abd EL Samea², Mohga S Abdalla ${ }^{3}$
}

\begin{abstract}
Objective: Cancer treatment using a targeted inducer of apoptosis like tumor necrosis factor-related apoptosisinducing ligand (TRAIL) faced the obstacle of resistance, thus providing a plus drug like Thymoquinone (TQ) could be of great interest to tackle breast cancer cells. The aim of the present work is to examine the genetic modulation impacts of the TRAIL receptors and apoptotic markers upon the combinatorial remedy of TRAIL plus TQ on human breast cancer cell lines. Methods: To achieve this rationale, the protein content-based cytotoxicity using SRB assay, as well as the genetic expressions of the TRAIL receptors (DR4 and DR5) and apoptotic markers (Bcl-2, Cas-8, and FADD) using real time qRT-PCR technique were preceded against breast cancer MCF-7 and MDA-MB-231 cancerous cell lines. Results: The current study showed that the combination therapy of TQ+TRAIL significantly inhibited the protein content-based proliferation of MDA-MB-231 cells more than MCF-7 cells. The synergistic effect of them significantly up-regulated the genetic expressions of DR4,DR5, Cas-8, and FADD genes and inhibited the genetic expression of the $B c l-2$ gene in the proposed cell lines treated for $24 \mathrm{~h}$. The induction of the apoptotic genes using the combined therapy was stimulated by the elevation of the reactive oxygen species (ROS); nitric oxide (NO) and malondialdehyde (MDA) levels. Conclusions: The synergistic influence between TQ which induced the DR5 and TRAIL, facilitating the connection between TRAIL and its receptors on the cancerous cell membrane. Hence, the proposed combination therapy induced the ROS-mediated apoptotic stimulus.
\end{abstract}

Keywords: TRAIL- thymoquinone- DR5- apoptotic genes- breast cancer cell lines

Asian Pac J Cancer Prev, 22 (9), 2855-2865

\section{Introduction}

Breast cancer (BC) is the second leading cause of death in women, accounting for roughly $30 \%$ of new occurrences (Zeng et al., 2017). Breast, liver, and bladder cancers are the most common cancers in both men and women in Egypt, according to the publications archive. Females were more likely to have breast and hepatic cancers, while males were more likely to develop hepatic and bladder cancers (Kassem et al., 2019).

Human breast cancer MCF-7 and MDA-MB-231 cell lines provide suitable models for in vitro treatment of breast cancer. Ovarian estrogens are required for the onset and progression of human breast cancer. The MCF-7 breast cancer cell line possesses efficient oestrogen receptors (ER), but the MDA-MB-231 human breast cancer cell line lacks ER and is unresponsive to ER and anti-ER (Ren et al., 2018).

There are numerous community therapies for various malignancies that prevent cancer proliferation by interfering with particular particles that are beneficial to cancer growth. The selected treatments are based on a better understanding of molecular genetics in malignant cell progression, which is used in the development of desirable remedies (Mansoori et al., 2017).

One of these crucial medicines, TNF-related apoptosis-inducing ligand (TRAIL) (Rieger et al., 1999), is marketed to specifically induce apoptosis in malignant cells such as breast, bladder, liver, and lung (Turner et al., 2014; Szliszka et al., 2012). TRAIL is a cytokine that is generated by various organs and has anticancer effect; hence it is found in the milk of nursing mothers (Zauli et al., 2013). TRAIL has been implicated in the eradication of cancer and the halting of its spread (Allen et al., 2013).

TRAIL can attach to its receptors (DR4 and DR5), triggering apoptosis through the intracellular death domain (DD) (Gasparian et al., 2009). The apoptotic signalling pathway produced by TRAIL is mediated by TRAIL binding to its receptors (DR4 and/or DR5), followed by receptor clustering, and then recruitment of the 
Fas-associated protein with death domain (FADD). The death-inducing signalling complex (DISC), also known as the $1^{\text {st }}$ complex, is formed when this FADD recruits caspase- 8 . This is followed by mitochondrial malfunction and the release of a mitochondrial activator (Smac) (Du et al., 2000).

Because TRAIL administration is difficult due to the development of TRAIL resistance, TRAIL combos have been proposed for over fifteen years to generate synergism or sensitise resistant cancer cells. Chemotherapy, which is either from natural agents or produced in the lab, limits the spread of cancer cells by preventing DNA damage that recruits cancer cells and generating apoptotic signals or preventing premalignant cells from proliferating into malignancy (Sun et al., 2017).

Many medicinal herbs have natural components that may be used to cure a hazardous and fatal disease such as carcinoma. The use of a natural agent with no side effects is critical in the treatment of such cancers (RafieianKopaei et al., 2017).

Thymoquinone (TQ), a potential cancer chemotherapeutic candidate derived from dietary components, has been demonstrated to have a significant role in overcoming the resistance of several malignancies, including breast and liver cancers, in multiple in-vitro and in-vivo studies (LIU et al., 2017; Woo et al., 2012).

TQ, a key component of Nigella Sativa, has been demonstrated to have anticancer properties (Woo et al., 2012).

TQ can be utilised as an anti-proliferative, pro-apoptotic, anti-angiogenic, and anti-angiogenic agent, as well as a cell cycle arrest inducer, according to the molecular signals (Majdalawieh and Fayyad, 2016; ElKhoely et al., 2015). TQ induced apoptosis and inhibited growth in pancreatic cancer cells, according to previous research (Chehl et al., 2009). Others looked at whether TQ had an inhibitory effect on malignant cell growth in a variety of cell types (Chen et al., 2012).

Overall, in the current study, we investigated the effect of TQ and TRAIL either individually or in combination as anticancer agents through studying the genetic expressions of the TRAIL receptors (DR4 and DR5) and the apoptotic signals (Bcl-2, Cas-8, and FADD genes). In addition, the reactive oxygen species (ROS); nitric oxide (NO) and malondialdehyde (MDA) levels were tested upon treatments on both cancerous cell lines (MCF-7 and MDA-MB-231).

\section{Materials and Methods}

\section{Materials}

Thymoquinone was purchased from Santa Cruz Biotechnology. TNF-related apoptosis-inducing ligand (TRAIL) and sulforhodamine B (SRB) for cellular protein measurement was purchased from Sigma-Aldrich (St. Louis, MO, USA). Dulbecco's Modified Eagle Medium (DMEM), Roswell Park Memorial Institute (RPMI) 1640 medium, fetal bovine serum (FBS), penicillin/streptomycin (P/S), l-glutamine, trypsin/EDTA, phosphate-buffered saline (PBS), and Annexin V stain for apoptosis analysis were obtained from Life Technologies,
Gibco (Grand Island, NY, USA). Nitric oxide (NO) and malondialdehyde (MDA) were purchased from (BIODIAGNOSTIC, Egypt). RNA Purification kit and RevertAid First strand cDNA synthesis kit were purchased from (Thermo Fisher Scientific, USA). SensiFAST SYBR LO-ROX mix kits were purchased from (Sigma). Primers for FADD, Caspase 8, Bcl-2, DR4 and DR5 versus GAPDH as a house keeping gene were generated using Primer3 program and verified by NCBI/ Primer-BLAST.

\section{Cell Lines}

Human breast cancer MCF7 (Cat. No. HTB-22 ${ }^{\text {TM}}$ ) and MDA-MB-231 (Cat. No. HTB-26 ${ }^{\mathrm{TM}}$ ) cell lines were purchased from Cell Culture Department, The Egyptian Holding Company for Biological products and vaccines (VACSERA), Giza, Egypt which purchased them from American Type Culture Collection (ATCC).

In-vitro studies

Cell culture and maintenance

MDA-MB-231 breast cancerous cell line is listed among the estrogen (ER), progesterone (PR), and HER2 triple-negative metastatic breast cancer panel (ATCC ${ }^{\circledR}$ No. TCP-1002 ${ }^{\mathrm{TM}}$ ). Despite both MDA-MB-231 and MCF7 cell lines are breast cancer cell lines, MDA-MB-231 is "basal" type and triple negative and MCF7 is "luminal" type and ER and PR positive cell lines. Thus, these differences affect the drug sensitivity. Breast cells were cultured using Dulbecco's modified Eagle's medium (DMEM) and Roswell Park Memorial Institute (RPMI-1640) medium. All media were supplemented with $4.5 \mathrm{~g} / \mathrm{L}$ Glucose with L-Glutamine and 10\% fetal bovine serum (FBS). The cells were incubated in $5 \% \mathrm{CO}_{2}$ humidified at $37^{\circ} \mathrm{C}$ for growth maintenance.

\section{Cellular protein measurements}

The protein contents of the human breast cancerous MCF-7 and MDA-MB-231 cell lines up on treatments with TQ, TRAIL, TQ+TRAIL, and 5-FU were measured according to the previously optimized method (Ahmed et al., 2015). Basically, sulforhodamine B (SRB), the protein dye, binds electrostatically and $\mathrm{pH}$ dependent on protein basic amino acid residues of trichloroacetic acid-fixed MCF-7 and MDA-MB-231 cells. After incubation with the proposed treatments, cell monolayers are fixed with $10 \%$ (wt/vol) trichloroacetic acid and stained with the $\mathrm{SRB}$ for $30 \mathrm{~min}$, after which the excess dye is removed by washing repeatedly with $1 \%$ (vol/vol) acetic acid. The protein-bound dye is dissolved in $10 \mathrm{mM}$ Tris base solution and observed at $510 \mathrm{~nm}$ using a microplate ELISA reader. This experiments were repeated three times $(n=3)$ and the results represented a method for detection the cytotoxic effect on the proposed cancerous cells based on the cellular protein content.

\section{The half inhibitory concentration $\left(I C_{50}\right)$ of the cancerous} cellular protein

The half maximal inhibitory concentrations $\left(\mathrm{IC}_{50}\right)$ values which means that the concentrations of the used TQ, TRAIL, TQ+TRAIL, or 5 -FU inhibit $50 \%$ of cell viabilities-based cellular protein content using MCF-7 
and MDA-MB-231 cell lines, were got by plotting the percentages of cell viabilities versus the concentrations of the sample using polynomial concentration-response curve fitting models (OriginPro 8 software).

\section{Apoptosis assay}

Apoptosis analysis was performed after fluorescence labeling of the damaged cancerous MCF-7 and MDA-MB-231 cellular membrane with Annexin V stain after treatment with the $\mathrm{IC}_{50}$ dosage of the proposed TQ, TRAIL, TQ+TRAIL, or 5-FU. The apoptotic fluorescence analysis, using (BMG Labtech, Germany), was performed to test the apoptotic shift after TQ, TRAIL, TQ+TRAIL, or 5-FU against control. Finally, the apoptotic cells (\%) of the MCF-7 and MDA-MB-231 breast cancerous cells were analyzed and figured out.

\section{Measurements of stress markers}

Nitric oxide (NO): NO plays an important role in trigging cancerous cell apoptosis. NO is rapidly oxidized to nitrite and nitrate which are used to quantitate $\mathrm{NO}$ production. Briefly, the MCF-7 and MDA-MB-231 cells were cultured in 96-well plates at a density of $1 \times 10^{4}$ cells/well. In the second day, the $\mathrm{IC}_{50}$ dosage of the proposed TQ, TRAIL, TQ+TRAIL, or 5-FU was added in the media. Nitrate reductase was firstly used to converts nitrate to nitrite. Then, Griess reagent was used to converts nitrite to a deep purple azo compound. The amount of the azo chromophore accurately reflected NO amount in the samples. Finally, optical density was measured at OD $540 \mathrm{~nm}$ using the microplate reader (BMG Labtech, Germany).

Malondialdehyde (MDA): Lipid peroxidation refers to the oxidative degradation of the cellular membrane lipids (to confirm annexin V-based apoptosis). In this process free radicals take electrons from the lipids in cell membranes, resulting in MCF-7 and MDA-MB-231 cells damage. The end product of lipid peroxidation is MDA. Briefly, the cells were cultured in 96-well plates at a density of $1 \times 10^{4}$ cells/well. In the second day, the $\mathrm{IC}_{50}$ dosage of the proposed TQ, TRAIL, TQ+TRAIL, or 5-FU was added in the media. The free MDA present in the sample was reacted with Thiobarbituric Acid (TBA) to generate a MDA-TBA adduct, which can be easily quantified colorimetrically at OD $532 \mathrm{~nm}$ using the microplate reader (BMG Labtech, Germany).

\section{Real Time qRT-PCR}

Total RNA Extraction: Total RNA was extracted from untreated control cells as well as treated MCF-7 and MDA-MB-231 cells with the $\mathrm{IC}_{50}$ dosage of the proposed TQ, TRAIL, TQ+TRAIL, or 5-FU using the Invitrogen RNA Purification kit (Thermo Fisher) according to the manufacturer's protocol. The concentration and the purity of RNA were assessed by Nanodrop Technologies at 260/280 ratio.

Conversion of RNA to cDNA: First-strand cDNA was synthesized with $1 \mu \mathrm{g}$ of total RNA using a RevertAid First strand cDNA synthesis kit (Thermo Fisher Scientific, USA) in accordance with the manufacturer's instructions. These samples were subsequently frozen at a temperature of $-80^{\circ} \mathrm{C}$ until use for determination of the expression levels of (FADD, Caspase 8, Bcl-2, DR4 and DR5) genes using real-time PCR.

\section{Real Time PCR reactions}

Quantitative real-time PCR was performed on Stratagene (Agilent) Mx3005PTM using the SensiFAST SYBR LO-ROX mix kits in addition to the forward and reverse primers for each gene. The nucleic acid sequences of the primers were as follows: Bcl-2 (forward: 5'-CTGCACCTGACGCCCTTCACC-3'\& reverse: 5'-CACATGACCCCACCGAACTCAAAGA-3'), c a s p a s e 8 ( f o r w a r d : 5, AGAGTCTGTGCCCAAATCAAC-3'\& reverse 5' - GCTGCTTCTCTCTTTGCTGAA- 3'), FADD (forward: 5'- CTCAGGTCCTGCCAGATGAAC-3' \& reverse: 5'-GGACGCTTCGGAGGTAGATG-3'), DR4 (forward: 5' -TCCAGCAAATGGTGCTGAC-3' \& reverse: 5'-GAGTCAAAGGGCACGATGTT-3'), DR5 (forward: 5'-CCAGCAAATGAAGGTGATCC-3' \& reverse: 5'-GCACCAAGTCTGCAAAGTCA-3') compared to $\beta$-actin as a housekeeping gene (forward: 5'-TTGCCGACAGGATGCAGAA-3' \& reverse: 5'GCCGATCCACACGGAGTACT -3').

Real-time PCR mixture consisted of $10 \mu \mathrm{L} 2 \mathrm{x}$ SensiFAST SYBR LO-ROX PCR Master Mix, $1 \mu \mathrm{L}$ of each primer $(400 \mathrm{nM}), 1 \mu \mathrm{L}$ cDNA, and $7 \mu \mathrm{L}$ Rnasefree water in a total volume of $20 \mu \mathrm{L}$. Amplification conditions and cycle counts were a temperature of $95^{\circ} \mathrm{C}$ for $15 \mathrm{~min}$ for the initial activation, followed by 40 cycles of denaturation at $95^{\circ} \mathrm{C}$ for $30 \mathrm{~s}$, annealing at $55^{\circ} \mathrm{C}$ for $30 \mathrm{~s}$, and elongation at $72^{\circ} \mathrm{C}$ for $30 \mathrm{~s}$. Melting curves were performed after real-time PCR to demonstrate the specific amplification of single products of interest. A standard curve assay was performed to determine the amplification efficiency of the primers used. Relative fold changes in the expression of target genes were accomplished using the comparative $2^{-\Delta \Delta \mathrm{Ct}}$ with the $\beta$-actin gene as an internal control to normalize the level of target gene expression. $\Delta \Delta \mathrm{CT}$ is the difference between the mean $\Delta \mathrm{CT}$ (treatment group) and mean $\Delta \mathrm{CT}$ (control group), where $\Delta \mathrm{CT}$ is the difference between the mean $C T$ gene of interest and the mean CT internal control gene in each sample. Logarithmic transformation was performed on fold change values before being statistically analyzed, using the fold change values of three replicates for each gene measured.

\section{Statistical analysis}

All experiments were carried out in three independent tests $(n=3)$. Data were expressed as the mean \pm standard deviation (SD) and analyzed using one-way analysis of variance (ANOVA). The results were considered statistically significant at probability $<0.05$.

\section{Results}

Cellular protein-based cytotoxicity and the $I C_{50}$

The purpose of this study was to use the SRB assay to assess the cellular protein content of MCF-7 and MDA-MB-231 cells. As a combinatorial anticancer drug, this reflects the cytotoxic action of TRAIL and TQ. The 
Table 1. MCF-7 Cellular Protein Measurements Using SRB Assay

\begin{tabular}{lcccc}
\hline $\begin{array}{l}\text { Treatment } \\
\text { Conc } \mu \mathrm{M}, \mathrm{nM}\end{array}$ & $\begin{array}{c}\text { TQ } \\
\text { Mean } \pm \mathrm{SD}\end{array}$ & $\begin{array}{c}\text { TRAIL } \\
\text { Mean } \pm \text { SD }\end{array}$ & $\begin{array}{c}\text { TQ+TRAIL } \\
\text { Mean } \pm \text { SD }\end{array}$ & $\begin{array}{c}5 \text {-FU } \\
\text { Mean } \pm \mathrm{SD}\end{array}$ \\
\hline 0 (Control) & $100 \pm 5$ & $100 \pm 5$ & $100 \pm 5$ & $100 \pm 5$ \\
20 & $82.81 \pm 4.14$ & $83.50 \pm 4.17$ & $52.16 \pm 2.60$ & $92.79 \pm 4.64$ \\
40 & $57.90 \pm 2.89$ & $58.59 \pm 2.93$ & $27.25 \pm 1.36$ & $67.88 \pm 3.39$ \\
60 & $55.42 \pm 2.77$ & $53.91 \pm 2.69$ & $25.18 \pm 1.25$ & $65.81 \pm 3.29$ \\
80 & $49.29 \pm 2.46$ & $48.33 \pm 2.41$ & $16.99 \pm 0.85$ & $56.54 \pm 2.82$ \\
100 & $47.33 \pm 2.36$ & $47.33 \pm 2.36$ & $13.71 \pm 0.68$ & $46.33 \pm 2.31$ \\
\hline
\end{tabular}

TQ, Thymoquinone; 5-FU, 5-Flurouracil; Control, untreated cancerous MCF-7 cells; SRB, sulforhodamine B; SD, standard deviation

anticancer capability of TRAIL and/or TQ against human breast cancer cell lines reflects the cells' normal function, and so cytotoxicity and cell viability are measured.

We initially studied the effect of TQ on the breast cancer cell death to investigate the effects of TQ on cancer cell growth, which showed that TQ can decrease the cell growth of both MDA-MB-231 and MCF-7 cancerous cells (Tables 1, 2). Using 5-Flurouracil (5-FU) as a positive control anti-cancer drug, the used 5-FU concentrations in the study were as follows: 0 (untreated cells), 20, 40, $60,80,100 \mu \mathrm{M}$ in parallel with the proposed TQ in $\mu \mathrm{M}$ unit, but the concentrations of TRAIL were the same but in $\mathrm{nM}$ unit.

There were a gradual and significant decrease ( $\mathrm{p}$ less than 0.05 ) the cancerous cell viability using SRB in both MCF-7 and MDA-MB-231 cancerous cells upon TQ treatment. In details, there was a slight cellular protein reduction effect of TQ on MCF-7 breast cancerous cells at $20 \mu \mathrm{M}$, where the cell viability was $82.816 \pm 4.14 \%$ after $24 \mathrm{~h}$. We found significant reduction effects of TQ upon MCF-7 breast cancerous cells until reached 47.331 $2.36 \%$ cell viability at $100 \mu \mathrm{M}$ after $24 \mathrm{~h}$ (Table 1 ), with the half maximal inhibitory doses $\left(\mathrm{IC}_{50}\right)$ value of TQ upon MCF-7 breast cancerous cells equal 69.306 \pm 3.46 $\mu \mathrm{M}$ (Table 3).

For MDA-MB-231 cancerous cells, there was a slight cellular protein reduction effect of TQ at $20 \mu \mathrm{M}$, where the cell viability was $60.124 \pm 3.00 \%$ after $24 \mathrm{~h}$. We found significant reduction effects of TQ upon MDA-MB-231 breast cancerous cells until reached $46.563 \pm 2.32 \%$ cell viability at $100 \mu \mathrm{M}$ after $24 \mathrm{~h}$ (Table 2), with the half maximal inhibitory concentrations $\left(\mathrm{IC}_{50}\right)$ value of TQ upon MDA-MB-231 breast cancerous cells equal 54.327士 $2.71 \mu \mathrm{M}$ (Table 3).
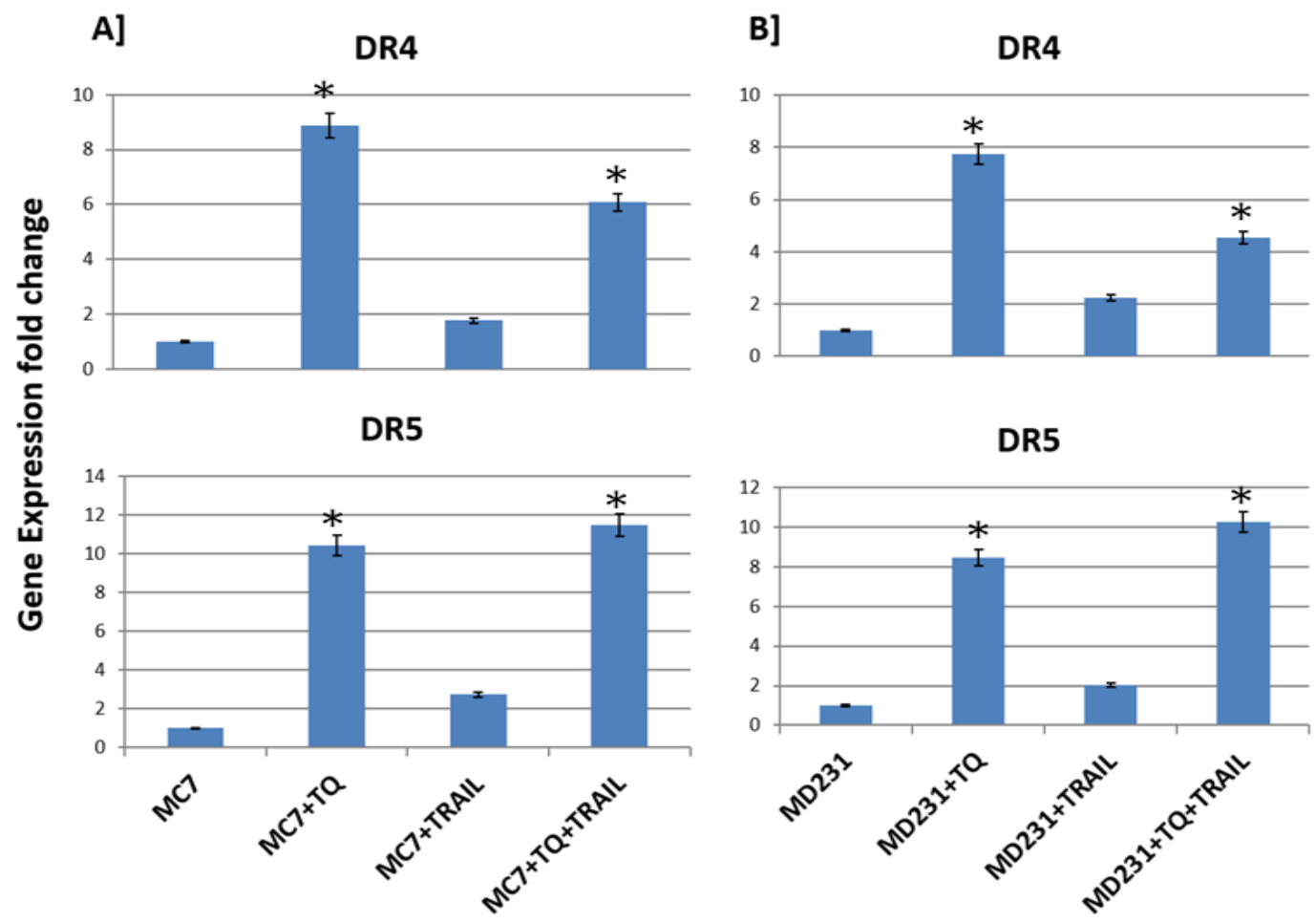

Figure 1. Genetic Expressions of the TRAIL Receptors. The individual TQ was firstly used to significantly upregulate the expressions of TRAIL-receptors, especially DR5, allowing the TRAIL subsequently to bind its DR receptors on the cancerous cell membranes, interfering its expression, so that the TRAIL receptors were not significantly upregulated. Collectively, both TQ+ TRAIL significantly induce the upregulation of TRAIL receptors, especially the DR5 TRAIL receptor. *; means high significance (p less than 0.01 ) when comparing treated cells with untreated cells. 
Table 2. MDA-MB-231 Cellular Protein Measurements Using SRB Aassay

\begin{tabular}{lcccc}
\hline $\begin{array}{l}\text { Treatment } \\
\text { Conc } \mu \mathrm{M}, \mathrm{nM}\end{array}$ & $\begin{array}{c}\text { TQ } \\
\text { Mean } \pm \text { SD }\end{array}$ & $\begin{array}{c}\text { TRAIL } \\
\text { Mean } \pm \text { SD }\end{array}$ & $\begin{array}{c}\text { TQ+TRAIL } \\
\text { Mean } \pm \text { SD }\end{array}$ & $\begin{array}{c}\text { 5-FU } \\
\text { Mean } \pm \text { SD }\end{array}$ \\
\hline 0 (Control) & $100 \pm 5$ & $100 \pm 5$ & $100 \pm 5$ & $100 \pm 5$ \\
20 & $60.12 \pm 3.00$ & $85.14 \pm 4.25$ & $43.51 \pm 2.17$ & $100.45 \pm 5.02$ \\
40 & $55.15 \pm 2.75$ & $74.86 \pm 3.74$ & $28.15 \pm 1.40$ & $75.32 \pm 3.76$ \\
60 & $55.88 \pm 2.79$ & $55.95 \pm 2.79$ & $25.29 \pm 1.26$ & $75.51 \pm 3.77$ \\
80 & $48.72 \pm 2.43$ & $39.06 \pm 1.95$ & $10.94 \pm 0.54$ & $57.54 \pm 2.87$ \\
100 & $46.56 \pm 2.32$ & $21.06 \pm 1.05$ & $1.96 \pm 0.09$ & $44.76 \pm 2.23$ \\
\hline
\end{tabular}

TQ, Thymoquinone; 5-FU, 5-Flurouracil; Control, untreated cancerous MDA-MB-231 cells; SRB, sulforhodamine B; SD, standard deviation

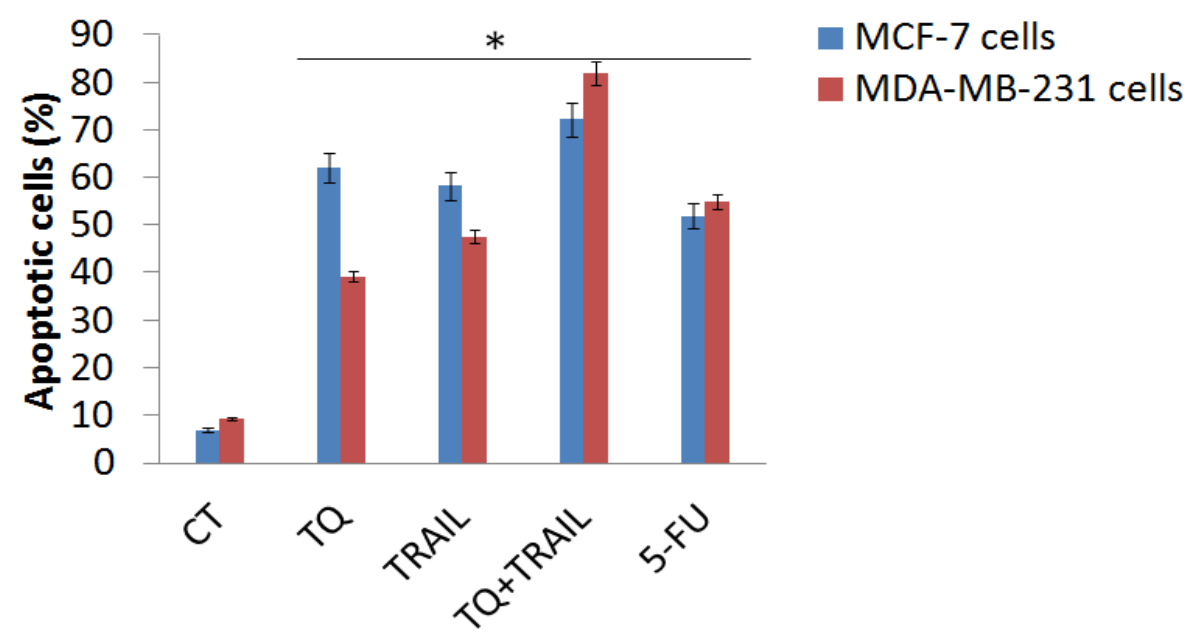

Treatments

Figure 2. Apoptotic Cancerous MCF-7 and MDA-MB-231 Cells (\%). The apoptotic positive shifts of the apoptotic cancerous MCF-7 and MDA-MB-231 cells (\%) were showed upon the proposed treatment, with the highest significance when using TQ+TRAIL combination therapy ( $\mathrm{p}$ less than 0.01 ). *; means high significance ( $\mathrm{p}$ less than 0.01 ) when comparing treated cells with control.

There were a gradual and significant decrease ( $\mathrm{p}$ less than 0.05 ) the cancerous cell viability using SRB in both MCF-7 and MDA-MB-231 cancerous cells upon TRAIL treatment. In details, there was a slight cellular protein reduction effect of TRAIL on MCF-7 breast cancerous cells at $20 \mu \mathrm{M}$, where the cell viability was $83.505 \pm 4.17$ $\%$ after $24 \mathrm{~h}$. We found significant reduction effects of TRAIL upon MCF-7 breast cancerous cells until reached $47.337 \pm 2.36 \%$ cell viability at $100 \mu \mathrm{M}$ after $24 \mathrm{~h}$ (Table $1)$, with the half maximal inhibitory concentrations $\left(\mathrm{IC}_{50}\right)$ value of TRAIL upon MCF-7 breast cancerous cells equal $67.489 \pm 3.37 \mu \mathrm{M}$ (Table 3).

For MDA-MB-231 cancerous cells, there was a slight cellular protein reduction effect of TRAIL at 20 $\mu \mathrm{M}$, where the cell viability was $85.140 \pm 4.25 \%$ after
$24 \mathrm{~h}$. We found significant reduction effects of TRAIL upon MDA-MB-231 breast cancerous cells until reached $21.063 \pm 1.05 \%$ cell viability at $100 \mu \mathrm{M}$ after $24 \mathrm{~h}$ (Table 2 ), with the half maximal inhibitory concentrations $\left(\mathrm{IC}_{50}\right)$ value of TRAIL upon MDA-MB-231 breast cancerous cells equal 68.090 $3.40 \mu \mathrm{M}$ (Table 3).

There were a gradual and highly significant decrease ( $p$ less than 0.01) the cancerous cell viability using SRB in both MCF-7 and MDA-MB-231 cancerous cells upon TQ+TRAIL treatment. In details, there was a slight cellular protein reduction effect of TQ+TRAIL on MCF-7 breast cancerous cells at $20 \mu \mathrm{M}$, where the cell viability was $52.163 \pm 2.60 \%$ after $24 \mathrm{~h}$. We found significant reduction effects of TQ+TRAIL upon MCF-7 breast cancerous cells until reached $13.714 \pm 0.68 \%$ cell viability at 100

Table 3. The IC50 of the Cellular Protein of MCF-7 and MDA-MB-231 Cell Lines

\begin{tabular}{lcccc}
\hline $\begin{array}{l}\text { Treatments } \\
\text { Cell lines }\end{array}$ & TQ & TRAIL & TQ+TRAIL & 5-FU \\
Mean \pm SD & Mean \pm SD & Mean \pm SD & Mean \pm SD \\
\hline MCF-7 & $69.30 \pm 3.465$ & $67.48 \pm 3.374$ & $26.04 \pm 1.302$ & $92.23 \pm 4.61$ \\
MDA-MB-231 & $54.32 \pm 2.716$ & $68.09 \pm 3.405$ & $25.17 \pm 1.259$ & $92.43 \pm 4.62$ \\
\hline
\end{tabular}

TQ, Thymoquinone; 5-FU, 5-Flurouracil; SD, standard deviation; IC50, The half maximal inhibitory concentrations values which means that the concentrations of the used TQ, TRAIL, TQ+TRAIL, or 5-FU inhibit 50\% of cell viabilities-based cellular protein content using MCF-7 and MDAMB-231 cell lines. 
A]

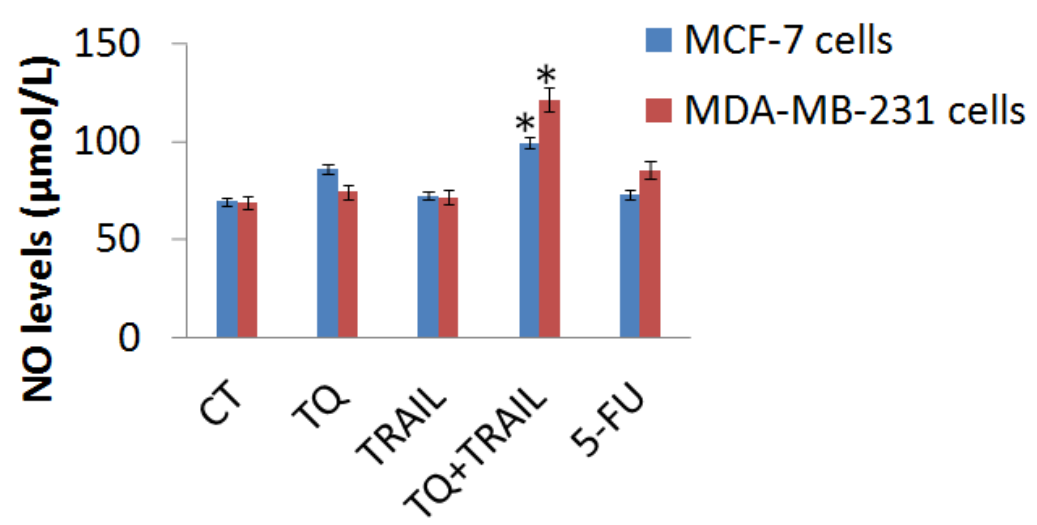

B]

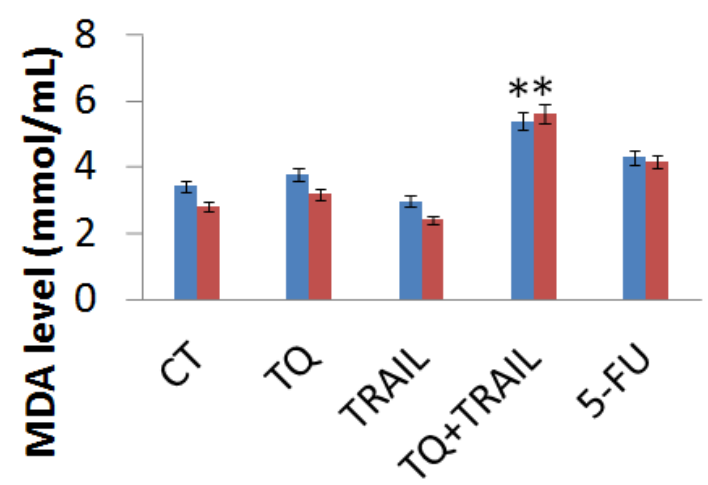

Treatments

Figure 3. Stress Markers Levels in the Cancerous MCF-7 and MDA-MB-231 Cells. A] Nitric oxide NO levels were measured upon treatments with TQ, TRAIL, TQ+TRAIL, and 5-FU. B] Malondialdehyde MDA levels were measured upon treatments with TQ, TRAIL, TQ+TRAIL, and 5-FU. The combination therapy of TQ+TRAIL showed the highest significance* of the NO and MDA levels (p less than 0.01 ).

$\mu \mathrm{M}$ after $24 \mathrm{~h}$ (Table 1), with the half maximal inhibitory concentrations $\left(\mathrm{IC}_{50}\right)$ value of TQ+TRAIL upon MCF-7 breast cancerous cells equal $26.045 \pm 1.30 \mu \mathrm{M}$ (Table 3).

For MDA-MB-231 cancerous cells, there was a slight cellular protein reduction effect of TQ+TRAIL at $20 \mu \mathrm{M}$, where the cell viability was $43.517 \pm 2.17 \%$ after $24 \mathrm{~h}$. We found significant reduction effects of TQ+TRAIL upon MDA-MB-231 breast cancerous cells until reached 1.963 \pm $0.09 \%$ cell viability at $100 \mu \mathrm{M}$ after $24 \mathrm{~h}$ (Table 2 ), with the half maximal inhibitory concentrations $\left(\mathrm{IC}_{50}\right)$ value of TQ+TRAIL upon MDA-MB-231 breast cancerous cells equal 25.171 $\pm 1.25 \mu \mathrm{M}$ (Table 3 ).

For 5-FU positive anti-cancer drug, there were a gradual and significant decrease ( $p$ less than 0.05) the cancerous cell viability using SRB in both MCF-7 and MDA-MB-231 cancerous cells upon 5-FU treatment. In details, there was a slight cellular protein reduction effect of 5-FU on MCF-7 breast cancerous cells at $20 \mu \mathrm{M}$, where the cell viability was $92.791 \pm 4.64 \%$ after $24 \mathrm{~h}$. We found significant reduction effects of 5-FU upon MCF-7 breast cancerous cells until reached $46.337 \pm 2.31 \%$ cell viability at $100 \mu \mathrm{M}$ after $24 \mathrm{~h}$ (Table 1 ), with the half maximal inhibitory concentrations $\left(\mathrm{IC}_{50}\right)$ value of 5-FU upon MCF-7 breast cancerous cells equal 92.231 \pm 4.61 $\mu \mathrm{M}$ (Table 3).

For MDA-MB-231 cancerous cells, there was a slight cellular protein reduction effect of 5-FU at 20 $\mu \mathrm{M}$, where the cell viability was $100.459 \pm 5.02 \%$ after $24 \mathrm{~h}$. We found significant reduction effects of 5-FU upon MDA-MB-231 breast cancerous cells until reached $44.763 \pm 2.23 \%$ cell viability at $100 \mu \mathrm{M}$ after $24 \mathrm{~h}$ (Table 2 ), with the half maximal inhibitory concentrations ( $\mathrm{IC}_{50}$ ) value of 5-FU upon MDA-MB-231 breast cancerous cells equal $92.439 \pm 4.62 \mu \mathrm{M}$ (Table 3 ).

\section{TRAIL-receptor genes}

In the current study, we showed that the TQ sensitized TRAIL-mediated apoptosis via up-regulation of TARIL receptors, especially DR5. These results provide the evidence that combined treatment with TQ may be a novel therapeutic approach for the successful TRAIL-based cancer.

Regarding TRAIL-receptors story, the individual TQ was firstly used to significantly upregulate the expressions of TRAIL-receptors, especially DR5, allowing the TRAIL subsequently to bind its DR receptors on the cancerous cell membranes, interfering its expression, so that the TRAIL receptors were not significantly upregulated. Collectively, both TQ+TRAIL significantly induce the upregulation of TRAIL receptors, especially the DR5 TRAIL receptor. These results happened up on treatment of breast cancer MCF-7 and MDA-MB-231 cell lines incubated for $24 \mathrm{~h}$ 
[A
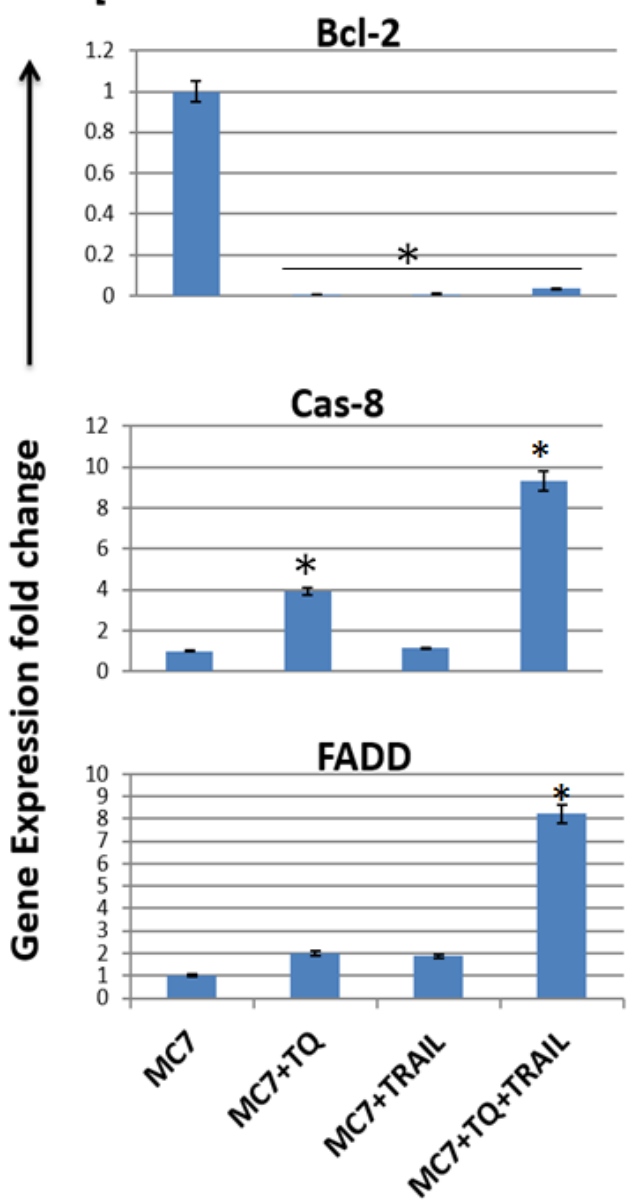

[B
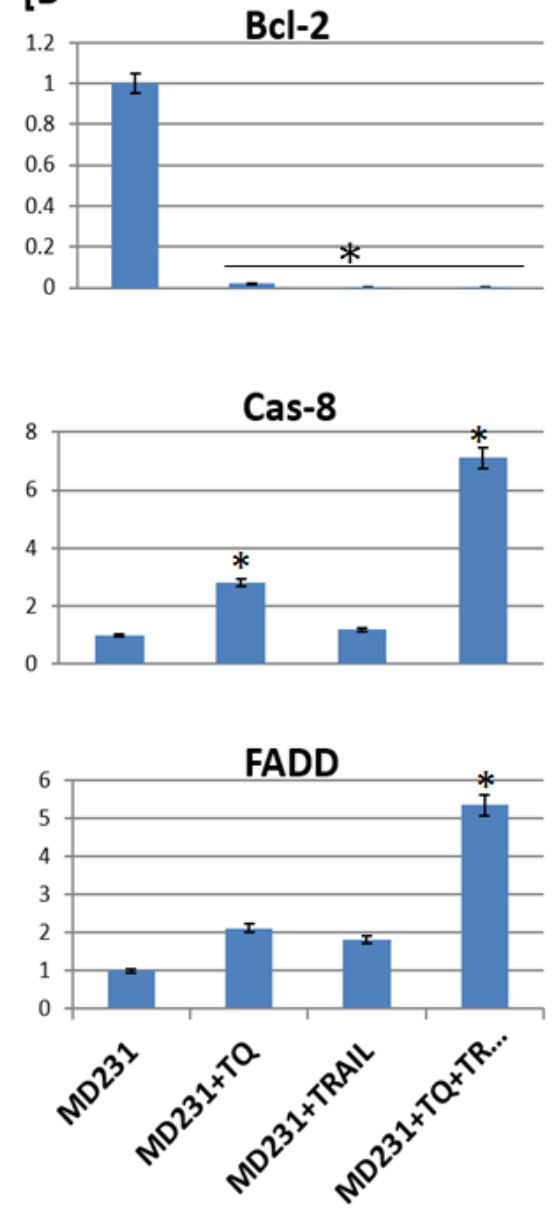

Figure 4. Genetic Expressions of the Apoptotic Genes. TQ and/or TRAIL inhibit the genetic expressions of $B c l-2$ gene and upregulate the genetic expressions of $C a s-8$ and FADD genes up on breast cancer MCF-7 (A) and MDA-MB-231 (B) cell lines treated for $24 \mathrm{~h}$ with noted TQ and/or TRAIL concentrations. The amounts of Bcl-2, Cas-8 and FADD genes were quantified using qRT-PCR and normalized to $\beta$-actin housekeeping gene. *; means high significance (p less than 0.01) when comparing treated cells with untreated cells.

with noted TQ and/or TRAIL concentrations. The genetic levels of DR4 and DR5 genes were quantified using real time $\mathrm{qRT}-\mathrm{PCR}$ and normalized to $\beta$-actin housekeeping gene (Figure 1).

TQ increased the expression of TRAIL receptors, although at different potencies. TQ was relatively more potent inducer of TRAIL-R2 (DR5) than TRAIL-R1 (DR4) (Figure 1). However, both death receptors were up-regulated by the TQ. This clearly suggests that TRAIL receptors, at least partly, contribute to TQ-induced MDA-MB-231 and MCF-7 cell inhibition by likely activating the extrinsic pathway of apoptosis in TQ-treated MDA-MB-231 and MCF-7 cell lines.

These results happened up on treatment of breast cancer MCF-7 and MDA-MB-231 cell lines incubated for $24 \mathrm{~h}$ with noted TQ and/or TRAIL concentrations. The genetic levels of the $D R 4$ and $D R 5$ genes were quantified using qRT-PCR and normalized to $\beta$-actin housekeeping gene.

\section{Apoptosis shifting}

We next determined the mechanism by which TQ and TRAIL kill MCF-7 and MDA-MB-231 breast cancerous cell lines. We observed the highest significant enhancement of apoptosis ( $p$ less than 0.01) of MDA-MB-231 cells treated with TQ+TRAIL (82.0\% apoptosis versus 9.45 $\%$ of the untreated control) compared with the individual treatments with TRAIL and TQ (Figure 2). In MCF-7 cell line, we found that the combination therapy of TQ+TRAIL induced $72.3 \%$ apoptosis versus $7.0 \%$ of the untreated control.

In addition, it was found that the individual treatments with TQ and TRAIL in MCF-7 and MDA-MB-231 breast cancerous cell lines represented a lower significant increase in apoptosis compared to the combinational therapy of them. Intriguingly, the apoptosis was induced more in MCF-7 breast cancerous cell line using TQ and TRAIL compared to MDA-MB-231 breast cancerous cell line (Figure 2).

For the standard anti-cancer 5-FU treatment, its induction of apoptosis was significantly less than our combinational therapy with TQ+TRAIL, where it we observed a significant enhancement of apoptosis ( $\mathrm{p}$ less than 0.01 ) of MDA-MB-231 cells treated with 5-FU ( $55.0 \%$ apoptosis versus $9.45 \%$ of the untreated control) compared with the individual treatments with TRAIL 
and TQ (Figure 2). In MCF-7 cell line, we found that the combination therapy of 5-FU induced $52 \%$ apoptosis versus $7.0 \%$ of the untreated control.

Overall, the apoptotic positive shifts of the apoptotic cancerous MCF-7 and MDA-MB-231 cells (\%) were showed upon the proposed treatment, with the highest significance with TQ+TRAIL combination therapy ( $p$ less than 0.01).

\section{TQ+TRAIL induce NO/MDA homeostasis}

Fig. 3 shows the NO (Figure 3A) and MDA (Figure 3B) levels produced by MCF-7 and MDA-MB-231 cells up on treatments with TQ, TRAIL, TQ+TRAIL, and 5-FU versus control. Both oxidative stress markers (NO and MDA) were high significantly increased (P less than 0.01) up on the combinational treatment with TQ+TRAIL compared to the untreated control, while MDA was slightly abolished up on MCF-7 and MDA-MB-231 cells treatment with TRAIL alone. Then, their levels were lucidity statistical elevated (P less than 0.01 ) up on its combination with TQ compared to control. Intriguingly, the combination of both TQ+TRAIL re-balance the MDA and NO levels in MCF-7 and MDA-MB-231 cells, making this therapeutic combinatorial formula specific to kill breast cancer MCF-7 and MDA-MB-231 cells. On the other hand, the standard anti-cancer 5-FU agent induced also the oxidative stress markers (NO and MDA) in both cancerous cell lines, but in a minor effect (P less than 0.05) compared to our combinatorial therapy with TQ+TRAIL (P less than 0.01).

\section{Apoptotic Genes}

Total RNA was extracted from control, treated MCF-7 and MDA-MB-231 cells. The concentration and the purity of RNA were assessed by Nano drop Technologies at 260/280 ratio and by gel electrophoresis, respectively.

Relative fold changes in the expression of target genes were accomplished using the comparative $2^{-\Delta \Delta \mathrm{Ct}}$ with the $\beta$-actin gene as an internal control to normalize the level of target gene expression. $\Delta \Delta \mathrm{CT}$ is the difference between the mean $\Delta \mathrm{CT}$ (treatment group) and mean $\Delta \mathrm{CT}$ (control group), where $\Delta \mathrm{CT}$ is the difference between the mean $\mathrm{CT}$ gene of interest and the mean $C T$ internal control gene in each sample. Logarithmic transformation was performed on fold change values before being statistically analysed, using the fold change values of three replicates for each gene measured.

We examined whether TQ regulated expression of the anti-apoptotic gene $\mathrm{Bcl}-2$. Real time Q-PCR analysis showed that TQ treatment at the $\mathrm{IC}_{50}$ for $24 \mathrm{~h}$ down-regulated mRNA level expression of the anti-apoptotic $\mathrm{Bcl}-2$ gene in MDA-MB-231 and MCF-7 cells. TQ treatments caused decreased the expression of Bcl-2 (Figure 4), indicating TQ-induced MDA-MB-231 and MCF-7 cells growth suppersion. Our RT-PCR results intriguingly revealed that TQ enhanced RNA levels of the pro-apoptotic genes Cas- 8 and FADD (Figure 4 ), both effects are known to target cells to apoptosis, would suggest that even in the presence of antagonistic effect of Bcl2 increase, the net effect of TQ presence is likely increased mitochondrial, intrinsic apoptosis in the MDA-MB-231 and MCF-7 cells.

The synergistic effect of the combination therapy of TQ and TRAIL significantly inhibit the genetic expressions of $B c l 2$ gene and upregulate the genetic expressions of Cas-8 and FADD genes up on breast cancer MCF-7 and MDA-MB-231 cell lines treated for $24 \mathrm{~h}$ with noted TQ or/and TRAIL concentrations. The amounts of $\mathrm{Bcl} 2$, Cas- 8 and $F A D D$ genes were quantified in triplicate $(\mathrm{n}=3)$ using qRT-PCR and normalized to $\beta$-actin housekeeping gene (Figure 4).

\section{Discussion}

The scarcity of death receptors expressed on the cell surface, and hence the lack of efficient targeting of these cells by TRAIL/agonistic mAb, limited the TRAILinduced apoptosis method. Furthermore, the emergence of TRAIL resistance has unfavourable consequences for TRAIL therapy (Mahalingam et al., 2009). However, the second mitochondrial activator of caspases, Smac, which is produced from mitochondria during the development of pro-apoptosis, can counteract this impact (Kaufmann et al., 2012).

Faced with the development of resistance to TRAILtargeted cell death, a new strategy has been devised in which TRAIL is paired with other medications (such as Thymoquinone TQ) that are more effective than TRAIL alone. Thymoquinone (TQ) is a potential natural chemical with considerable anticancer activity in vitro and in vivo against a variety of malignant cell types (Sarmistha et al., 2018). It is a potent inducer of cell cycle arrest and apoptosis. Preclinical studies reveal the potential of TQ in improving the therapeutic effect of anticancer drugs and also protection of non-tumor tissues against chemotherapy-induced damages (El-Baba et al., 2014).

The primary goal of combinatorial-based TRAIL is to either synergize TRAIL activity or sensitise TRAIL-resistant cells to TRAIL. To that purpose, most combinatorial techniques aimed at synergizing TRAIL and/or sensitising resistant tumours to TRAIL include chemical and natural substances (Zaidi et al., 2009).

In parallel with the current study, the combination therapy of TQ+TRAIL had a gradual and highly significant decrease upon the cancerous protein content (i.e. cell viability) using SRB in both MCF-7 and MDA-MB-231 cells. The synergistic effect of this combinatorial therapy reported an $\mathrm{IC}_{50}$ value upon $\mathrm{MCF}-7$ breast cancerous cells equal $26.045 \pm 1.30 \mu \mathrm{M}$ and an $\mathrm{IC}_{50}$ value upon MDA-MB-231 breast cancerous cells equal $25.171 \pm$ $1.25 \mu \mathrm{M}$.

Intriguingly, the combination therapy of TQ+TRAIL reported lower $\mathrm{IC}_{50}$ values compared to the standard 5-FU positive anti-cancer drug, which recorded an $\mathrm{IC}_{50}$ value upon MCF-7 cells equal 92.231 $\pm 4.61 \mu \mathrm{M}$ and an $\mathrm{IC}_{50}$ value upon MDA-MB-231 breast cells equal $92.439 \pm 4.62 \mu \mathrm{M}$. These findings validated the promising anticancer effect of TQ+TRAIL in breast cancer MCF-7 and MDA-MB-231 cells based on protein content (SRB test), which is consistent with our recently published paper that found similar results but used mitochondrial 
MTT labelling instead of SRB staining (Abd El-Salam et al., 2021).

In the Middle East, thymoquinone, a bioactive component of Nigella sativa, is widely used (Badary et al., 2001). It contains antioxidant properties and has been found in animal experiments to protect against heart, liver, and kidney damage (Badary et al., 1997). TQ has been shown to decrease cell proliferation in a variety of cancer cells, including breast, colon, and lung cancer cells, in recent research. Previous research has demonstrated that TQ's cytotoxic effects are limited to cancer cells (Banerjee et al., 2010). We reviewed the effects of TQ on the growth of breast cancer MCF-7 and MDA-MB-231 cells, as well as the genetic signals of TRAIL receptors (DR4 and DR5) and apoptotic genes. In the case of TRAIL-receptors, the chemotherapy TQ was used to significantly upregulate the expressions of TRAIL-receptors, particularly DR5, allowing TRAIL to bind to its DR receptors on cancerous cell membranes, interfering with their expression, resulting in the TRAIL receptors not being significantly upregulated. Both TQ and TRAIL together dramatically increase TRAIL receptor expression.

These findings came from the treatment of $\mathrm{MCF}-7$ and MDA-MB-231 breast cancer cell lines. Combinatorial methods mostly work via endoplasmic reticulum (ER) stress, resulting in overexpression of DR5 and/or DR4 and increased TRAIL-induced apoptosis (Refaat et al., 2014). As a result, we examined stress markers (ROS) and discovered that the combined therapy with TQ+TRAIL remarkably induced the NO and MDA levels in the breast cancerous cells.

The activation of CHOP via p38/ERK MAPKs, which increases DR5 transcription, is another downstream checkpoint (Woo et al., 2013). Up-regulating pro-apoptotic genes (Ghosh et al., 2012) or downregulating anti-apoptotic genes (Martn-Pérez et al., 2012), both of which are in perfect agreement with the current study's findings.

These previous results were in parallel with our results regarding the $D R 4$ and $D R 5$ genetic expression levels after the combination of TRAIL and TQ treatment, where in the current study, we showed that the TQ sensitizes TRAIL-mediated apoptosis via up-regulation of DR4 and DR5 as well as the enrolment of the ROS markers (NO and MDA). These results provide the evidence that combined treatment with TQ may be a novel therapeutic approach for the successful TRAIL-based cancer therapy.

To further elucidate molecular mechanisms of apoptosis signalling by TQ, we next determined alterations in levels of pro- and anti-apoptotic genes using qRT-PCR. Specifically, we examined expression of the pro-apoptotic genes and TRAIL receptors in TQ-treated breast cancer cell lines, MDA-MB-231 and MCF7 cells. TQ increased the expression of TRAIL receptors, although at different potencies. TQ was relatively more potent inducer of TRAIL-R2 (DR5) than TRAIL-R1 (DR4), as it up-regulated the expression of DR5, while DR4 expression was slightly affected. However, both death receptors were up-regulated by higher TQ concentrations. This clearly suggests that TRAIL receptors, at least partly, contribute to TQ-induced breast cancerous cell growth inhibition by likely activating the extrinsic pathway of apoptosis in TQ-treated cells. Our data support recent findings showing that DR5 (TRAIL-R2) is selectively up-regulated by TQ in human cancer cell lines (Hussain et al., 2011). Interestingly, Choi and companions (Choi et al., 2002) reported that DR5 ligation by TRAIL not only leads to apoptosis of human glioma cells but also induces expression of IL-8. Therefore, the TQ and TRAIL not only induce cancer cell death mediated by DR 5 but also induce immune cell mediation.

The apoptotic ligand TRAIL binds to death domaincontaining cell surface receptors, resulting in a connection between TRAIL receptors (DR4 and DR5) and apoptotic genes (Rahman et al., 2009). Despite promising data indicating that TRAIL produced apoptosis in a large number of malignant cells, about half of cancer cell cultures were resistant to TRAIL-mediated cell death (Singh et al., 2003), as we reported in our study. Remarkably, up-regulation of DR4, DR5 and pro-apoptotic genes, as well as down-regulation of the anti-apoptotic B-cell lymphoma 2 ( $\mathrm{Bcl}-2)$ gene and inhibitors of apoptosis in TRAIL-resistant tumor cell lines correlated with sensitization to TRAIL-induced apoptosis (Singh et al., 2003; Poulain et al., 2009), which in-line with our genetic profiling results.

TQ treatment for 24 hours reduced the mRNA level expression of the anti-apoptotic B-cell lymphoma 2 (Bcl-2) gene in MDA-MB-231 and MCF-7 breast cancer cells, according to real-time qRT-PCR analysis. These findings suggested that TQ inhibited the proliferation of MDA-MB-231 and MCF-7 cells. TQ's downregulation of Bcl-2 is accompanied by an increase in DR5 (Gali Muhtasib et al., 2004; Liet al., 2011; Vikhanskaya et al., 2007).

The real time qRT-PCR results intriguingly revealed that the combination therapy of TQ+TRAIL significantly decreased the expression of the anti-apoptotic gene $\mathrm{Bcl}-2$ and increased expression of the pro-apoptotic genes Cas-8 and FADD, both effects are known to target cells to apoptosis, the net effect of the combination therapy is increased the mitochondrial, intrinsic apoptosis in the MDA-MB-231 and MCF-7cells as in discussed in previous publications (Abd El-Salam et al., 2021; Oltvai et al., 1993; LaCasse et al., 1998).

Collectively, we may conclude that the combination of TRAIL and TQ have highly increased expression of the pro-apoptotic genes Cas- 8 and FADD, both effects are known to target cells to higher apoptosis mediated by the increment of DR5 gene and ROS (NO and MDA) levels.

\section{Author Contribution Statement}

All authors contributed in this work. HMI Sharada, GG Abd EL Samea, MS Abdalla, and AA Abd-Rabou are the supervisors of the NM Abdel Salam's PhD thesis. AA Abd-Rabou had the work idea and was responsible for paper writing, editing, and publishing it, with the help of NM Abdel Salam. NM Abdel Salam and AA Abd-Rabou performed the cell culture, biochemical, and genetic experiments. HMI Sharada, GG Abd EL Samea, and MS Abdalla shared in all experiments and edited the manuscript.

Asian Pacific Journal of Cancer Prevention, Vol 22 


\section{Acknowledgements}

We would like to acknowledge Egyptian Company for Blood Transfusion Services (EgyBlood), VACSERA, Faculty of Science, Helwan University, Egypt, and the National Research Center, for facilitating this work.

\section{Funding statement}

The Egyptian Company for Blood Transfusion Services (EgyBlood), VACSERA shared in funding the $\mathrm{PhD}$ thesis of NM Abdel Salam.

The authors declare that this paper is a part of NM Abdel Salam's PhD thesis.

\section{Ethical Approval}

This paper as a part of NM Abdel Salam's PhD thesis which was ethically approved from the Egyptian Company for Blood Transfusion Services (EgyBlood), VACSERA, and Faculty of Science, Helwan University, Egypt.

\section{Availability of data}

Available after the approval of all authors.

\section{Competing Interests}

The authors declare that there is no conflict of interest.

\section{References}

Abdel Salam NM, Abd-Rabou A A, Sharada H I, Abd EL Samea G G, Abdalla M S (2021). Combination therapy of TRAIL and Thymoquinone induce breast cancer cell cytotoxicitymediated apoptosis and cell cycle arrest. Asian Pac J Cancer Biol, 22, 1513-21.

Allen JE, Krigsfeld G, Mayes PA, et al (2013). Dual inactivation of Akt and ERK by TIC10 signals Foxo3a nuclear translocation, TRAIL gene induction, and potent antitumor effects. Sci Transl Med, 5, 171ra17.

Badary OA, Gamal El-Din AM (2001). Inhibitory effects of thymoquinone against 20-methylcholanthrene-induced fibrosarcoma tumorigenesis. Cancer Detect Prev, 25, 362-8.

Badary OA, Nagi MN, al-Shabanah OA, et al (1997). Thymoquinone ameliorates the nephrotoxicity induced by cisplatin in rodents and potentiates its antitumor activity. Can J Physiol Pharmacol, 75, 1356-61.

Banerjee S, Padhye S, Azm A, et al(2010). Review on molecular and therapeutic potential of thymoquinone in cancer. Nutr Cancer, 62, 938-46.

Chehl N, Chipitsyna G, Gong Q C J, Yeo H A Arafat (2009). Anti-inflammatory effects of the Nigella sativa seed extract, thymoquinone, in pancreatic cancer cells. $H P B, \mathbf{1 1}, 373-81$.

Chen KF, Chen HL, Liu CY, et al (2012). Dovitinib sensitizes hepatocellular carcinoma cells to TRAIL and tigatuzumAb, a novel anti-DR5 antibody, through SHP-1-dependent inhibition of STAT3. Biochem Pharmacol, 83, 769-77.

Choi C, Kutsch O, Park J, et al (2002). Tumor necrosis factorrelated apoptosis-inducing ligand induces caspase-dependent interleukin-8 expression and apoptosis in human astroglioma cells. Mol Cell Biol, 22, 724-36.

Du C, Fang M, Li Y, Li L, Wang X (2000). Smac, a mitochondrial protein that promotes cytochrome c-dependent caspase activation by eliminating IAP inhibition. Cell, 102, 33-42.

El-Baba C, Mahadevan V, Fahlbusch FB, et al (2014). Thymoquinone-induced conformational changes of PAK1 interrupt prosurvival MEK-ERK signaling in colorectal cancer. Mol Cancer, 13, 201.

ElKhoely A, Hafez HF, Ashmawy AM, et al (2015). Chemopreventive and therapeutic potentials of thymoquinone in HepG2 cells. mechanistic perspectives. J Nat Med, 2015.

Gali Muhtasib H, Diab-Assaf M, Boltze C, et al (2004). Thymoquinone extracted from black seed triggers apoptotic cell death in human colorectal cancer cells via a p53-dependent mechanism. Int J Oncol, 25, 857-66.

Gasparian ME, Chernyak BV, Dolgikh DA, et al (2009). Generation of new TRAIL mutants DR5-A and DR5-B with improved selectivity to death receptor 5 . Apoptosis. Int $J$ Program Cell Death, 14, 778-87.

Ghosh AP, Klocke BJ, Ballestas ME, Roth KA (2012). CHOP potentially co-operates with FOXO3a in neuronal cells to regulate PUMA and BIM expression in response to ER stress. PLoS One, 2012.

Hussain AR, Ahmed M, Ahmed S, et al (2011). Thymoquinone suppresses growth and induces apoptosis via generation of reactive oxygen species in primary effusion lymphoma. Free Radical Biol Med, 50, 978-87.

Kassem MN, Makar SM, Kassem HA, et al (2019). Circulating miR-34a and miR-125b as promising non-invasive biomarkers in Egyptian locally advanced breast cancer patients. Asian Pac J Cancer Prev, 20, 2749-55.

Kaufmann T, Strasser A, Jost PJ (2012). Fas death receptor signalling: roles of Bid and XIAP. Cell Death Differ, 19, 42-50.

LaCasse EC, Baird S, Korneluk RG, MacKenzie AE (1998). The inhibitors of apoptosis (IAPs) and their emerging role in cancer. Oncogene, 17, 3247-59.

Li Q, Zhao LY, Zheng Z, et al (2011). Inhibition of p53 by adenovirus type $12 \mathrm{E} 1 \mathrm{~B}-55 \mathrm{~K}$ deregulates cell cycle control and sensitizes tumor cells to genotoxic agents. J Virol, 2011.

Liu S, Ou S, Huang H (2017). Green tea polyphenols induce cell death in breast cancer MCF-7 cells through induction of cell cycle arrest and mitochondrial-mediated apoptosis. $J$ Zhejiang Univ-Sci B (Biomedicine \& Biotechnology), 18, 89-98.

Mahalingam D, Szegezdi E, Keane M, de Jong S, Samali A (2009). TRAIL receptor signalling and modulation: Are we on the right TRAIL?. Cancer Treat Rev, 35, 280-8.

Majdalawieh AF, Fayyad MW (2016). Recent advances on the anti-cancer properties of Nigella sativa, a widely used food additive. Ayurveda Integr Med, 7, 173-80.

Mansoori B, Mohammadi A, Davudian S, Shirjang S, Baradaran B (2017). The different mechanisms of cancer drug resistance. Adv Pharm Bull, 7, 339-48.

Martín-Pérez R, Niwa M, López-Rivas A (2012). ER stress sensitizes cells to TRAIL through down-regulation of FLIP and Mcl-1 and PERK-dependent up-regulation of TRAIL-R2. Apoptosis Int J Program Cell Death, 17, 349-63.

Oltvai ZN, Milliman CL, Korsmeyer SJ (1993). Bcl-2 heterodimerizes in vivo with a conserved homolog, Bax, that accelerates programmed cell death. Cell, 74, 609-19.

Poulain S, Evenou F, Carre MC, et al (2009). Vitamin A/retinoids signalling in the human lung. Lung Cancer, 66, 1-7.

Rafieian-Kopaei M, Movahedi M (2017). Breast cancer chemopreventive and chemotherapeutic effects of Camellia Sinensis (green tea). Electron Physician, 9, 3838-44.

Rahman M, Pumphrey JG, Lipkowitz S (2009). The TRAIL to targeted therapy of breast cancer. Adv Cancer Res, 103, 43-73.

Refaat A, Abd Rabou AA, Reda A (2014). TRAIL combinations: The new 'trail' for cancer therapy (Review). Oncol Lett, 7, 1327-32.

Ren G, Shi Z, Teng C, Yao Y (2018). Antiproliferative activity of combined biochanin a and ginsenoside Rh2 
on MDA-MB-231 and MCF-7 human breast cancer cells. Molecules, 23, 2908.

Rieger J, Ohgaki H, Kleihues P, Weller M (1999). Human astrocytic brain tumors express AP02L/TRAIL. Acta Neuropathol (Berl), 97, 1-4.

Singh TR, Shankar S, Chen X, Asim M, Srivastava RK (2003). Synergistic interactions of chemotherapeutic drugs and tumor necrosis factor-related apoptosis-inducing ligand/ Apo-2 ligand on apoptosis and on regression of breast carcinoma in vivo. Cancer Res, 63, 5390-5400.

Sun Y, Zhao Z, YangZ, et al (2017). Risk factors and preventions of breast cancer. Int J Biol Sci, 13, 1387-97.

Szliszka E, Czuba ZP, Kawczyk-Krupka A,et al (2012). Chlorinbased photodynamic therapy enhances the effect of tumor necrosis factor-related apoptosis-inducing ligand (TRAIL) in bladder cancer cells. Med Sci Monit Int Med J Exp Clin Res, 18, BR47-53.

Turner A, Li LC, Pilli T, Qian L, et al (2013). MADD knock-down enhances doxorubicin and TRAIL induced apoptosis in breast cancer cells. PLoS One, 2013.

Vikhanskaya F, Lee MK, Mazzoletti M, Broggini M, Sabapathy K (2007). Cancer-derived p53 mutants suppress p53-target gene expression-potential mechanism for gain of function of mutant p53. Nucleic Acids Res, 35, 2093-2104.

Woo CC, Kumar AP, Sethi G, TanK HB (2012). Thymoquinone: potential cure for inflammatory disorders and cancer. Biochem Pharmacol, 83, 443-51.

Woo JS, Kim SM, Jeong CH, Ryu CH, Jeun SS (2013). Lipoxygenase inhibitor MK886 potentiates TRAIL-induced apoptosis through CHOP- and p38 MAPK-mediated up-regulation of death receptor 5 in malignant glioma. Biochem Biophys Res Commun, 431, 354-9.

Zauli G, Monasta L, Rimondi E, et al (2013). Levels of TNFrelated apoptosis-inducing ligand (TRAIL) show a long-term stability in the breast milk of mothers of preterm infants. $J$ Hum Lact, 29, 350-3.

Zeng L, Yan J, Luo L, Ma M, Zhu H (2017). Preparation and characterization of Epigallocatechin-3-gallate (EGCG)loaded nanoparticles and their inhibitory effects on Human breast cancer MCF-7 cells. Sci Rep, 7, 45521.

Zhang M, Du H, Huang Z et al (2018). Thymoquinone induces apoptosis in bladder cancer cell via endoplasmic reticulum stress-dependent mitochondrial pathway. Chem Biol Interact, 292, 65-75.

\section{cc) (i) (8)}

This work is licensed under a Creative Commons AttributionNon Commercial 4.0 International License. 